\title{
A mathematical model of fracturing fluid leak-off based on dynamic discrete grid system
}

\author{
Yaokun Yang ${ }^{1,2} \cdot$ Hongwei Jiang ${ }^{1} \cdot \mathrm{Mu} \mathrm{Li}^{1,2} \cdot$ Shuai Yang ${ }^{1,2} \cdot$ Gang Chen $^{1,2}$
}

Received: 17 November 2014/Accepted: 31 May 2015/Published online: 23 June 2015

(C) The Author(s) 2015. This article is published with open access at Springerlink.com

\begin{abstract}
Based on the classical PKN two-dimensional fracture propagation mathematical model, the two-dimensional leak-off model of fracturing fluid of fractured dualmedium reservoir is established by considering the timevarying non-Newtonian fracturing fluid leak-off coefficient in the stretching process of fractures. Using the finite element difference method, a dynamic discrete grid system is established and solved by Newton-Raphson iterative method. At the same time, the effect on fracturing fluid leak-off of the fractured reservoir stress sensitivity coefficient, the pumping rate, and the propagating length of the fractures is analyzed. As it is analyzed, under the combined effect of the formation pressure, the fracture pressure, the edge effect, and the fracture permeability, the greater the stress sensitivity coefficient is, the smaller the leak-off rate and coefficient are. However, the greater pumping rate is, the larger leak-off rate and coefficient are. If both of them increase to a certain value, the leak-off coefficient firstly decreases, and then increases; the longer the fracture is, in the same position, the larger fracturing fluid leak-off coefficient is; and the greater boundary effect is, the larger fracturing fluid leak-off coefficient near the pinch point is.
\end{abstract}

Keywords Low-permeability reservoirs - Filtration coefficient $\cdot$ Fracture propagation - Stress sensitivity · Dynamic grid

Yaokun Yang

1228926825@qq.com

1 China National Petroleum Corporation Drilling Research Institute, Beijing 102206, China

2 College of Petroleum Engineering, China University of Petroleum, Beijing 102249, China

\section{Introduction}

Low-permeability reservoirs need to be developed with fracturing technology (Balen et al. 1988; Demarchos and Chomatas 2004; Fan and Economides 1995); due to the presence of natural fractures in the reservoirs, the conventional homogeneous reservoir fracturing fluid leak-off model is no longer applicable (Settari 1985; Yi and Penden 1993; Settari 1998). For the double-porosity reservoir fluid leak-off calculation model, many scholars have studied it. Considering single permeability and dual permeability (Mayerhofer et al. 1991; Nghiem et al. 1984), some established one-dimensional fracturing fluid leak-off models for fractured dual-medium rese rvoirs, and then considering the actual situation that fracturing fluid leak-off in the formation is two-dimensional flowing fluid and the fracturing fluid is non-Newtonian fluid, they established the two-dimensional model of non-Newtonian fracturing fluid leak-off, which improved the pressure fracturing fluid leak-off model, rendering the results more in line with the actual situation. However, the above models are established on the basis of the situation that the fractures do not extend after the pump stops and the pressure distributes evenly in the fracture, and combining the actual mineral conditions, the fracturing fluid leak-off also exists in the propagation process. For the propagation of the fractures, the classical two-dimensional PKN and KGD models and the pseudo-three dimensional (P3D) model are mainly used to conduct the simulation (AlShatri et al. 2009; Ouenes and Hartley 2000), and we can make the assumption that the fracturing fluid filtration coefficient is constant. However, the leak-off process does not accord with the classic Carter leak-off model. The actual filtration coefficient changes with time and is associated with the fluid flowing process among the 
formation and the fractures (Economides and Demarchos 2008; Gidley et al. 1989)).

At present, the main point is the stress sensitivity when the formation pressure decreases in production. Therefore, the fracturing fluid leak-off makes the reservoir pressure increase, and the permeability of the stress sensitive reservoir may increase. So it is necessary to discuss the increasing volume of leak-off fluid. This paper combines the dual-porosity formation flow equations with PKN two-dimensional fracture-stretching model, and considering the filtration coefficient dynamic variation during the fracture-stretching process, and the effect of the reservoir stress sensitivity, we will establish two-dimensional fracturing fluid leak-off model. Compared with the actual site, the results of simulation get better adaptability.

\section{Mathematical model}

\section{Formation}

According to the Darcy law, when fracturing fluid flows in low-permeability reservoirs fractures and matrix, the differential equations are derived asFlowing in the fractures:

$-\nabla \cdot\left(v_{f}\right)+\alpha \frac{K_{m}}{\mu_{e m}}\left(P_{m}-P_{f}\right)=\left(\phi C_{t}\right)_{f} \frac{\partial P_{f}}{\partial t}$

Flowing in the matrix:

$-\nabla \cdot\left(v_{m}\right)-\alpha \frac{K_{m}}{\mu_{e m}}\left(P_{m}-P_{f}\right)=\left(\phi C_{t}\right)_{m} \frac{\partial P_{m}}{\partial t}$.

Wherein, when the fracturing fluid is considered as nonNewtonian fluid, the equation of motion can be derived as

$v_{i}=-\frac{K_{i}}{\mu_{e i}} \nabla P_{i}, \quad(i=f, m)$

$\mu_{e i}=\frac{3 n+1}{8 n}\left(\frac{\phi_{i}}{8 K_{i}}\right)^{\frac{1-n}{2 n}}\left(2 k_{n}\right)^{\frac{1}{n}}\left(-\nabla P_{i}\right)^{\frac{n-1}{n}}, \quad(i=f, m)$.

For the reservoirs of which the depth is relatively shallow and the deformation of the skeleton particles is obvious, such as the coalbed methane reservoirs, when the fracturing fluid filtrates into the reservoirs, the formation pressure increases, which may cause fractures or matrix porosity to expand and the permeability to increase. For the low-permeability reservoir characteristics, only the stress sensitivity is considered, namelyInitial condition:

$K_{f}=K_{f i} e^{-\beta\left(P_{i}-P_{f}\right)}$

$P_{j}(x, y, 0)=P_{i}, \quad(j=f, m)$

Boundary condition

$$
\begin{aligned}
& \frac{K_{f}}{\mu_{e f}} \frac{\partial P_{f}}{\partial y}=\frac{C(x)}{\sqrt{t-\tau(x)}}, \quad(0<x<L(t), y=0) \quad \frac{\partial P_{f}}{\partial y}=0 \\
& \quad\left(L(t)<x<x_{e}, y=0\right) \\
& \frac{\partial P_{f}}{\partial y}=0,\left(0<x<x_{e}, y=y_{e}\right) \quad \frac{\partial P_{f}}{\partial x}=0 \\
& \quad\left(x=0,0<y<y_{e}\right) \\
& \frac{\partial P_{f}}{\partial x}=0, \quad\left(x=x_{e}, 0<y<y_{e}\right)
\end{aligned}
$$

\section{Artificial fractures}

The classic PKN two-dimensional model is used to simulate the propagation of fractures in the formation, and the fracture height is constantly equal to the effective reservoir thickness. While considering the fracturing fluid leaking off into the formation, the filtration coefficient is not the same at different locations and changes with time.

Considering the artificial fracture vertical face's strain and combining with the England and Green equation, we can derive the fracture width equation:

$W(x, t)=\frac{(1-v) H\left(P_{F}(x, t)-\sigma_{h}\right)}{G}$.

Considering the artificial fracture vertical profile as oval and the fracturing fluid as non-Newtonian fluid, when the fracturing fluid flows in the fracture, we can obtain the pressure drop equation as

$\frac{\partial P_{F}}{\partial x}=-\frac{64}{\pi} \frac{q}{W^{3} H} K_{n}\left(\frac{2 n+1}{3 n}\right)^{n}\left(\frac{6 q}{H W^{2}}\right)^{n-1}$.

when the fracturing fluid flows in the artificial fracture, the continuity equation can be expressed as

$\frac{\partial q}{\partial x}+\frac{\pi H}{4} \frac{\partial W}{\partial t}+\frac{2 C H}{\sqrt{t-\tau}}=0$

Initial condition:

$W(x, t)=0, \quad x>L(t)$

Boundary condition:

$W(x, 0)=0$

$\left(\frac{\partial W^{4}}{\partial x}\right)_{x=0}=\frac{256(1-v) Q}{\pi G}, \quad x=0$.

\section{The model solution}

\section{Formation}

Combined with Eqs. (1)-(5), and considering the quasi steady-state channeling in matrix and the two-dimensional flow of the fracturing fluid in the formation, the synthesis 


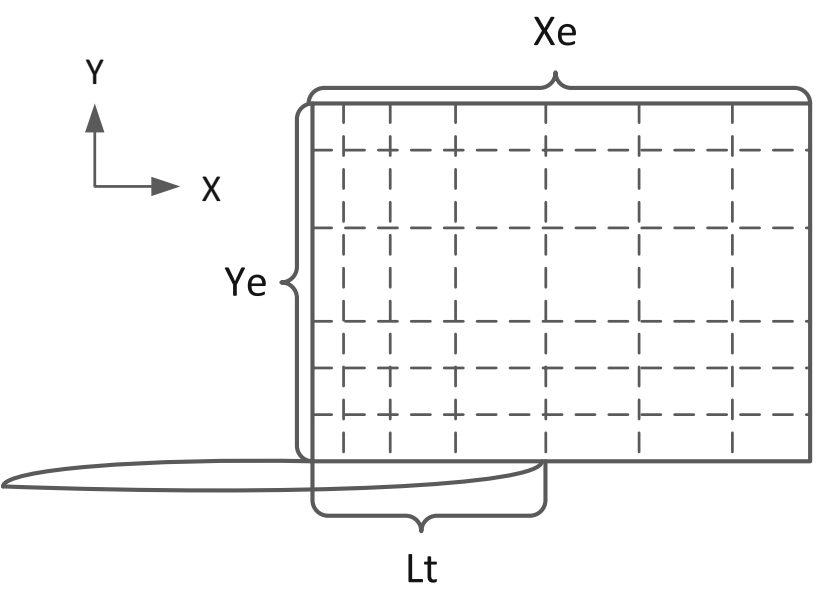

Fig. 1 Dynamic grid

flow equation in fracture and matrix can be derived asFracture:

$\nabla \cdot\left(\frac{K_{f i} e^{-\beta\left(P_{i}-P_{f}\right)}}{\mu_{e f}} \nabla P_{f}\right)+\alpha \frac{K_{m}}{\mu_{e m}}\left(P_{m}-P_{f}\right)=\left(\phi C_{t}\right)_{f} \frac{\partial P_{f}}{\partial t}$

Matrix:

$-\alpha \frac{K_{m}}{\mu_{e m}}\left(P_{m}-P_{f}\right)=\left(\phi C_{t}\right)_{m} \frac{\partial P_{m}}{\partial t}$.

Equation (15) shows a strong non-linear characteristic, and it is difficult to the analytical solution. Therefore, the finite-difference distribution combined with the NewtonRaphson iterative method is used to solve the equation. With the fracturing proceeding, the fractures keep propagating, which makes it difficult to divide meshes. So a dynamic discrete grid is established in Fig. 1, which works as follows: according to the increase of the fractures, the number of meshes is increased and all the time steps are assumed to be equal to $T_{0}$. During the first period $T_{0}$, the fracture length is $L_{\mathrm{F} 1}$, and the entire area is divided into the two grids in the fracture crack orientation. The length of the grids are, respectively, $L_{\mathrm{F} 1}$ and $\left(X_{\mathrm{e}}-L_{\mathrm{F} 1}\right)$. During the

Fig. 2 Dynamic grid schematic diagram in different time second period $T_{0}$, the length of the fracture increases by $L_{\mathrm{F} 2}$, and the entire region is divided into three grids in the direction of the fracture, and the length of the grids are, respectively, $L_{\mathrm{F} 1}, L_{\mathrm{F} 2}$, and $\left(X \mathrm{e}-L_{\mathrm{F} 1}-L_{\mathrm{F} 2}\right)$. We can find that the pressure of the grid of $L_{\mathrm{F} 2}$ during the last period is the pressure on the grid of $\left(X_{\mathrm{e}}-L_{\mathrm{F} 1}\right)$ during the last period. It can be successively obtained according to this method that during the $\mathrm{n}$ period of $T_{0}$, the length of the fracture increases by $L_{\mathrm{Fn}}$, and the whole region is divided into $(n+1)$ grids in the direction of the fracture, the length of which are, respectively, $L_{\mathrm{F} 1}, L_{\mathrm{F} 2}, \ldots, L_{\mathrm{Fn}}$, and $\left[\mathrm{Xe}-\left(L_{\mathrm{F} 1}\right.\right.$ $\left.\left.+L_{\mathrm{F} 2}+\cdots+L_{\mathrm{Fn}}\right)\right]$ and the pressure on the grid $\mathrm{L}_{\mathrm{Fn}}$ during this period is equal to the pressure on the last grid of $\left[\mathrm{Xe}-\left(L_{\mathrm{F} 1}+L_{\mathrm{F} 2}+\cdots+L_{\mathrm{Fn}-1}\right)\right]$ during last period. Dynamic meshes dividing diagram is shown as follows in Fig. 2:

Now the differential discretization is conducted for the formulas (15) and (16), which is the process of simple discretization. We regard the fracture and the matrix of channeling as the same grid, while we can make use of the pressure value of last period to calculate the effective crack permeability and effective viscosity of this period. Then we can get the final differential equation as follows:

$$
\begin{aligned}
& a_{i, j} P_{f(i, j-1)}^{N+1}+b_{i, j} P_{f(i-1, j)}^{N+1}+c_{i, j} P_{f(i, j)}^{N+1}+d_{i, j} P_{f(i+1, j)}^{N+1} \\
& \quad+e_{i, j} P_{f(i, j+1)}^{N+1}=g_{i, j} .
\end{aligned}
$$

Among them:

$$
\begin{gathered}
a_{i, j}=\frac{2 K_{f i} e^{-\beta\left(P_{i}-P_{f(i, j)}^{N}\right)}}{\Delta y_{j} \mu_{e f(j-1 / 2)}\left(\Delta y_{j}+\Delta y_{j-1}\right)} \\
b_{i, j}=\frac{2 K_{f i} e^{-\beta\left(P_{i}-P_{f(i, j)}^{N}\right)}}{\Delta x_{i} \mu_{e f(i-1 / 2)}\left(\Delta x_{i}+\Delta x_{i-1}\right)} \\
d_{i, j}=\frac{2 K_{f i} e^{-\beta\left(P_{i}-P_{f(i, j)}^{N}\right)}}{\Delta x_{i} \mu_{e f(i+1 / 2)}\left(\Delta x_{i}+\Delta x_{i+1}\right)} \\
e_{i, j}=\frac{2 K_{f i} e^{-\beta\left(P_{i}-P_{f(i, j)}^{N}\right)}}{\Delta y_{j} \mu_{e f(j+1 / 2)}\left(\Delta y_{j}+\Delta y_{j+1}\right)}
\end{gathered}
$$

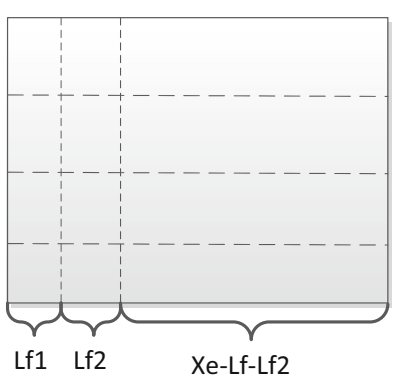

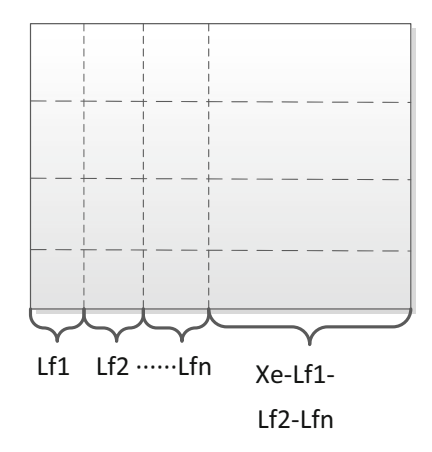

(3) $\mathrm{nT}_{0}$
(2) $2 \mathrm{~T}_{0}$

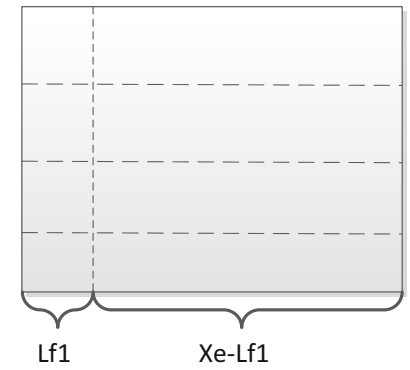

(1) $T_{0}$ 


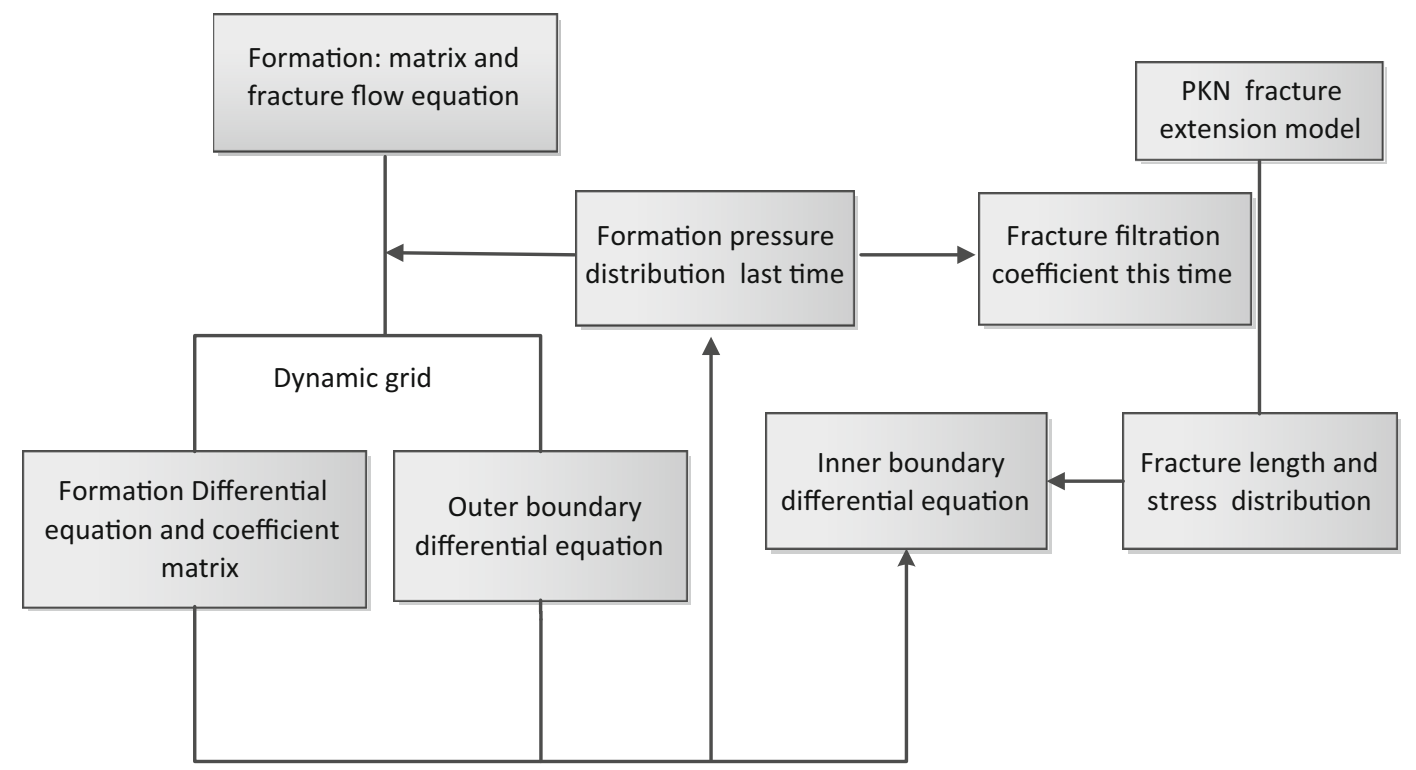

Fig. 3 Solving flowchart

$$
\begin{aligned}
& g_{i, j}=-\frac{\left(C_{t} \phi\right)_{f} P_{f(i, j)}^{N}}{T_{0}}-\frac{\left(C_{t} \phi\right)_{m} \alpha K_{m} P_{m(i, j)}^{N}}{\mu_{e m(i, j)}\left(C_{t} \phi\right)_{m}+\alpha T_{0} K_{m}} \\
& c_{i, j}=-\left(a_{i, j}+b_{i, j}+d_{i, j}+e_{i, j}\right)-\frac{\left(C_{t} \phi\right)_{f}}{T_{0}} \\
& -\frac{\left(C_{t} \phi\right)_{m} \alpha K_{m}}{\mu_{e m(i, j)}\left(C_{t} \phi\right)_{m}+\alpha T_{0} K_{m}} \\
& \mu_{e f(i \pm 1 / 2)}=\frac{3 n+1}{8 n}\left(\frac{\phi_{f}}{8 K_{f i} e^{-\beta\left(P_{i}-P_{f(i, j)}^{N}\right)}}\right)^{\frac{1-n}{2 n}} \\
& \times\left(2 k_{n}\right)^{\frac{1}{n}}\left|\frac{P_{f(i \pm 1)}^{N}-P_{f(i)}^{N}}{\left(\Delta x_{i}+\Delta x_{i \pm 1}\right) / 2}\right|^{\frac{n-1}{n}} \\
& \times c_{i, j}=-\left(a_{i, j}+b_{i, j}+d_{i, j}+e_{i, j}\right) \\
& -\frac{\left(C_{t} \phi\right)_{f}}{T_{0}}-\frac{\left(C_{t} \phi\right)_{m} \alpha K_{m}}{\mu_{e m(i, j)}\left(C_{t} \phi\right)_{m}+\alpha T_{0} K_{m}} \\
& \mu_{e m(i, j)}=\frac{3 n+1}{8 n}\left(\frac{\phi_{f}}{8 K_{f i} e^{-\beta\left(P_{i}-P_{m(i, j)}^{N}\right)}}\right)^{\frac{1-n}{2 n}} \\
& \times\left(2 k_{n}\right)^{\frac{1}{n}} \mid \frac{P_{m(i+1 j)}^{N}-P_{m(i-1, j)}^{N}}{\left(\Delta x_{i-1}+2 \Delta x_{i}+\Delta x_{i+1}\right)} \\
& +\left.\frac{P_{m(i, j+1)}^{N}-P_{m(i, j-1)}^{N}}{\left(\Delta y_{j-1}+2 \Delta y_{j}+\Delta y_{j+1}\right)}\right|^{\frac{n-1}{n}} .
\end{aligned}
$$

\section{Artificial fracture}

The fracture synthetical flowing equation can be derived of the combination from Eqs. (9) to (11) as

$\frac{G}{64(1-v) H \mu} \frac{\partial^{2} W^{4}}{\partial x^{2}}-\frac{\partial W}{\partial t}-\frac{8 C}{\pi \sqrt{t-\tau}}=0$.
On the basis of the value of the pressure distribution of last period, the value of the leak-off in Eq. (18) can be approximated as

$\frac{C}{\sqrt{t-\tau}}=\frac{2 K_{f i} e^{-\beta\left(P_{i}-P_{f(i, 1)}^{N}\right)}\left(P_{F}^{N}-P_{f(i, 1)}^{N}\right)}{\mu_{e f} \Delta y_{1}}$.

Thus, by the " $t$ " period (which can also be expressed as NT0), we can get the filtration coefficient at the fracture tip as

$C=\frac{2 K_{f i} e^{-\beta\left(P_{i}-P_{f(i, 1)}^{N}\right)}\left(P_{F}^{N}-P_{f(i, 1)}^{N}\right)}{\mu_{e f} \Delta y_{1}} \sqrt{N T_{0}}$.

With the combination of the Eqs. (18) and (20), according to Carter method, at the " $t$ " period (which can be expressed as the first $(N+1)$ one time T0), the full length and pressure distribution of the fracture are derived as

$$
\begin{aligned}
& L\left((N+1) T_{0}\right)=Q \sqrt{\frac{N+1}{N}} \frac{\mu_{e f} \Delta y_{1}}{2 K_{f i} e^{-\beta\left(P_{i}-P_{f(i, 1)}^{N}\right)}\left(P_{F}^{N}-P_{f(i, 1)}^{N}\right)} \\
& P_{F}=\frac{4 G}{(1-v) H}\left[\frac{2(1-v) \mu Q^{2}}{\pi^{3} G C H}\right]^{\frac{1}{4}} t^{\frac{1}{8}} \\
& \times\left\{\frac{x}{L} \sin ^{-1}\left(\frac{x}{L}\right)+\left[1-\left(\frac{x}{L}\right)^{2}\right]^{\frac{1}{4}}-\frac{\pi}{2} \frac{x}{L}\right\}^{\frac{1}{4}}+\sigma_{H} .
\end{aligned}
$$

Then the fracture is regarded as the boundary condition of formation pressure, which can be expressed as

$P_{f(i, 1)}^{N+1}=P_{F}^{N+1}-\frac{C \mu_{e f} \Delta y_{1}}{2 K_{f i} e^{-\beta\left(P_{i}-P_{f(i, 1)}^{N}\right)} \sqrt{(N+1) T_{0}}}$. 
Based on all the equations above, considering the additional boundary condition and initial condition, we can use the strong implicit Newton-Raphson iterative method to derive the pressure value at each point. By using the Eq. (20), we can get fracturing fluid filtration coefficient and rate at different locations. The overall calculation flowchart is shown as follows: first, we need to discrete the formation flowing equation, then combine it with the formation pressure distribution at last period to obtain the correlation coefficient and the outer boundary condition of differential equations; then we make use of PKN model and combine it with the pressure distribution of grids nearby the fracture on the last moment to derive the dynamic filtration coefficient, and the length of the fracture and pressure distribution in fractures are obtained. Then the pressure distribution of fractures is regarded as a boundary condition of the formation; the differential equations, the outer boundary conditions, and the inner boundary conditions are integrated to obtain the current reservoir pressure by using the Newton-Raphson iterative method and the fracturing fluid filtration coefficient is finally obtained. Solving flowchart is shown in Fig. 3.

\section{Calculation and analysis}

Based on the models above, making use of non-uniform grid mesh which is divided into 20 perpendicular to the fracture, we may analyze the examples and factors. The other basic data are shown in Table 1.

As is shown in Fig. 4, considering stress sensitivity during the fracture propagation process, we can get the law of fracturing fluid leak-off. It can be concluded from Fig. 4, as the stress sensitivity coefficients increase, the fracturing

Table 1 related basic data

\begin{tabular}{ll}
\hline Parameters & Value \\
\hline Well control area $\left(\mathrm{m}^{2}\right)$ & $\begin{array}{c}\text { xe }=100 \mathrm{~m}, \\
\text { ye }=100 \mathrm{~m}\end{array}$ \\
Initial formation pressure $(\mathrm{Mpa})$ & 25 \\
Initial fracture permeability, $\mathrm{Kfi}(\mathrm{mD})$ & 10 \\
Stress sensitivity coefficient, $\beta\left(\mathrm{MPa}^{-1}\right)$ & 0.5 \\
Minimum horizontal stress $(\mathrm{MPa})$ & 20.5 \\
Consistency coefficient, $\mathrm{Kn}(\mathrm{Pa} \mathrm{sn})$ & 0.01 \\
Fracture porosity, $\Phi_{\mathrm{f}}$ & 0.01 \\
Fluid index, $n$ & 0.85 \\
Matrix porosity, $\Phi \mathrm{m}$ & 0.01 \\
Matrix permeability, Km(mD) & 1 \\
Fracture compressibility coefficient, $\mathrm{Ctf}\left(\mathrm{MPa}^{-1}\right)$ & 0.00025 \\
Matrix compressibility coefficient Ctm $\left(\mathrm{MPa}^{-1}\right)$ & 0.00030 \\
Pumping volume, $\mathrm{Q}(\mathrm{m} /$ min $)$ & $10^{3}$ \\
\hline
\end{tabular}
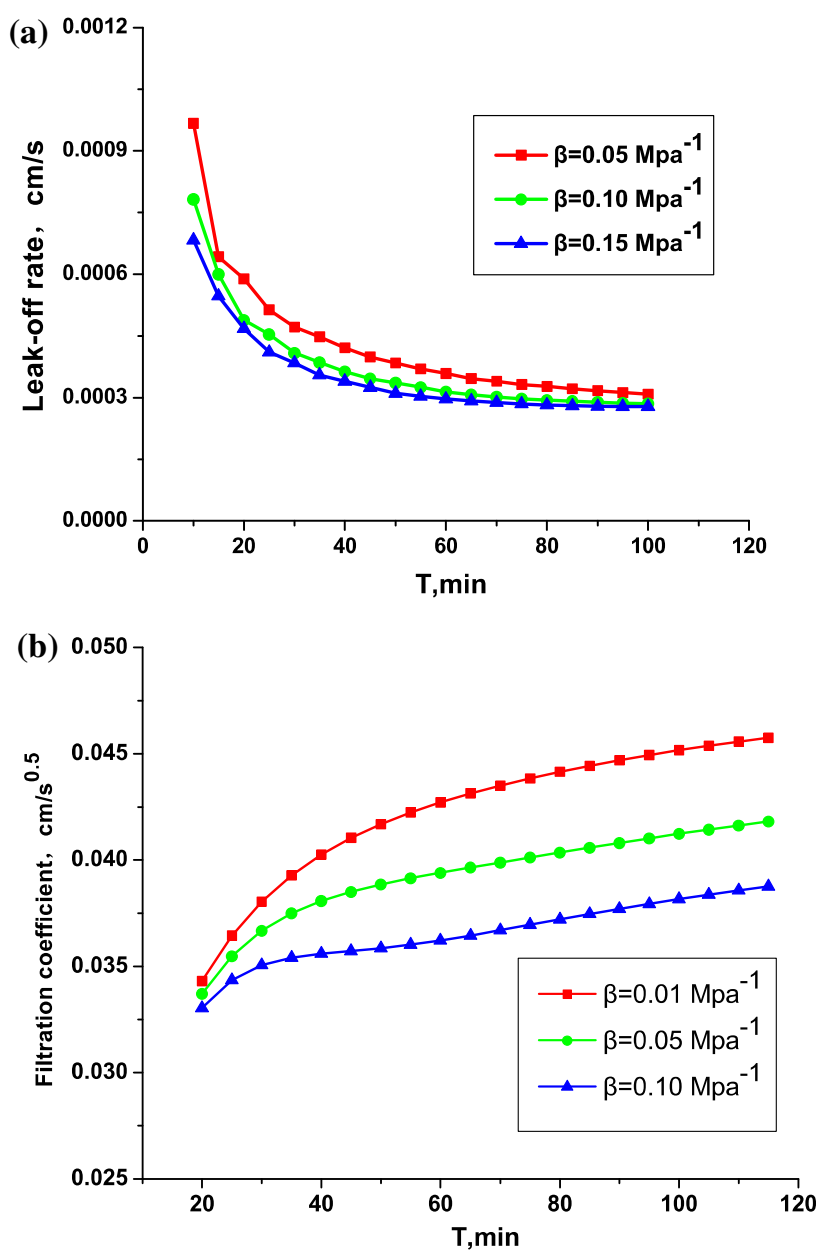

Fig. 4 The effect of stress sensitivity on fracturing fluid leak-off: a leak-off rate, $\mathbf{b}$ leak-off coefficient

fluid rate and filtration coefficient decrease; as time passes by, the leak-off rate decreases but the filtration coefficient increases. In the beginning of fracturing, both the fracturing fluid rate and filtration coefficient appear obvious variation, and both of them remain unchanged later. As is shown in Fig. $4 \mathrm{~b}$, when the stress sensitivity coefficient increases to a certain value, the filtration coefficient will firstly decreases and then increases. Based on comprehensive analysis of the reason, it is obtained that, along with the continuous injection of the fracturing fluid, the artificial fracture's pressure continues to increase, and due to the fracturing fluid leak-off and the increase of the formation pressure, the stress sensitivity makes the formation fractures open and the permeability increase, which improve the capacity of the increase of the formation pressure. Also as the fracture permeability increases, the comprehensive effect results in the decrease of leak-off. When the pressure wave reaches the boundary, the sealed boundary will weaken the effect; therefore, the fracturing fluid filtration coefficient will increase again. 

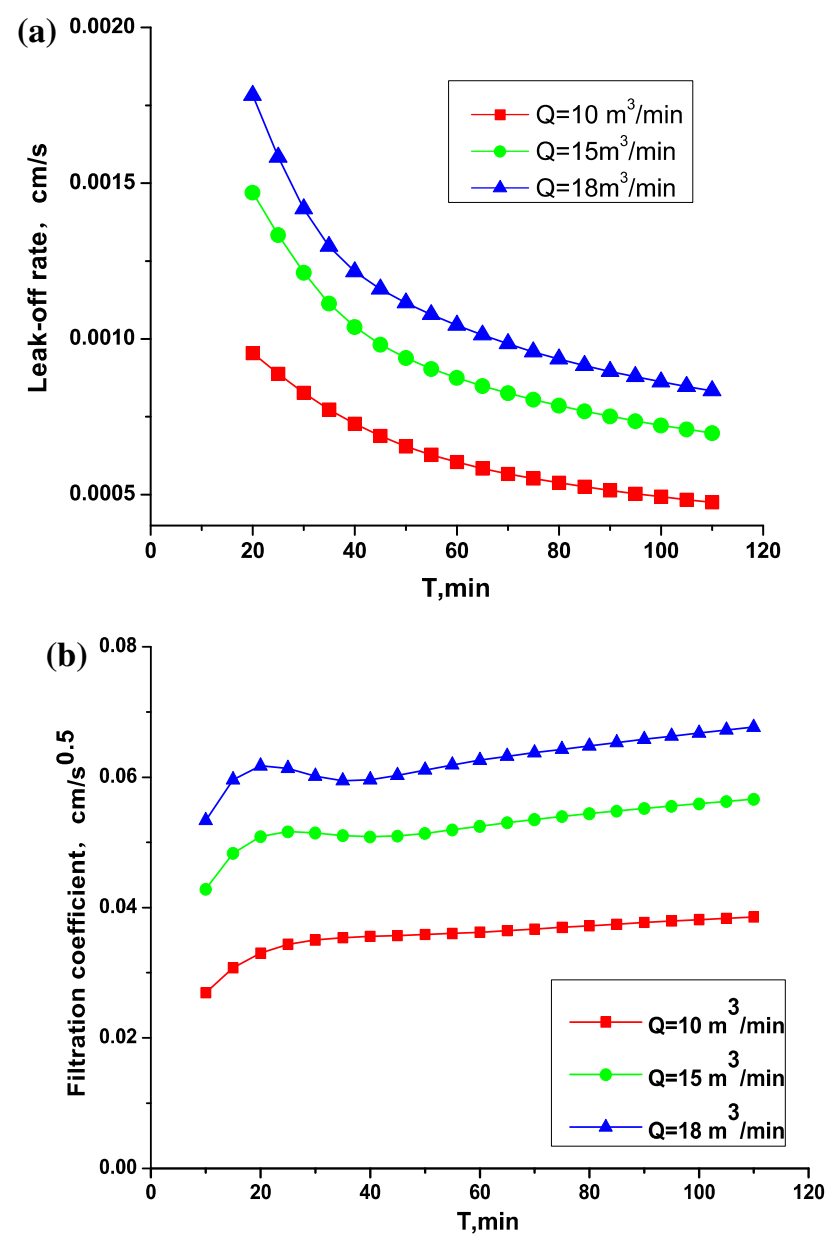

Fig. 5 The effect of pumping rate on fracturing fluid leak-off: a leakoff rate, b leak-off coefficient

Figure 5 shows the laws of fracturing fluid leak-off considering the different fracturing fluid pumping rate during the fracture propagation process. It can concluded from Fig. 5, as the fracturing fluid pumping rate increases, the leak-off velocity and filtration coefficient will increase. As time passes by, the leak-off rate decreases but the filtration coefficient continues to increase. In the beginning of the fracturing, both of them appear obvious variation first and remain unchanged later. As is shown in Fig. 4b, when the stress sensitivity coefficient increases to a certain value, the filtration coefficient will firstly decrease and then increase. Based on comprehensive analysis of the mechanism, the increased volume of fluid pumped in increases the fluid pressure of the fractures, and then increases the fracturing fluid leak-off. However, when the volume increases to a certain value, the formation pressure will increase, the fracture permeability resulting from the stress sensitivity will increase, and the artificial fracture pressure will also increase. This comprehensive effect makes the filtration coefficient decrease, and the sealed boundary
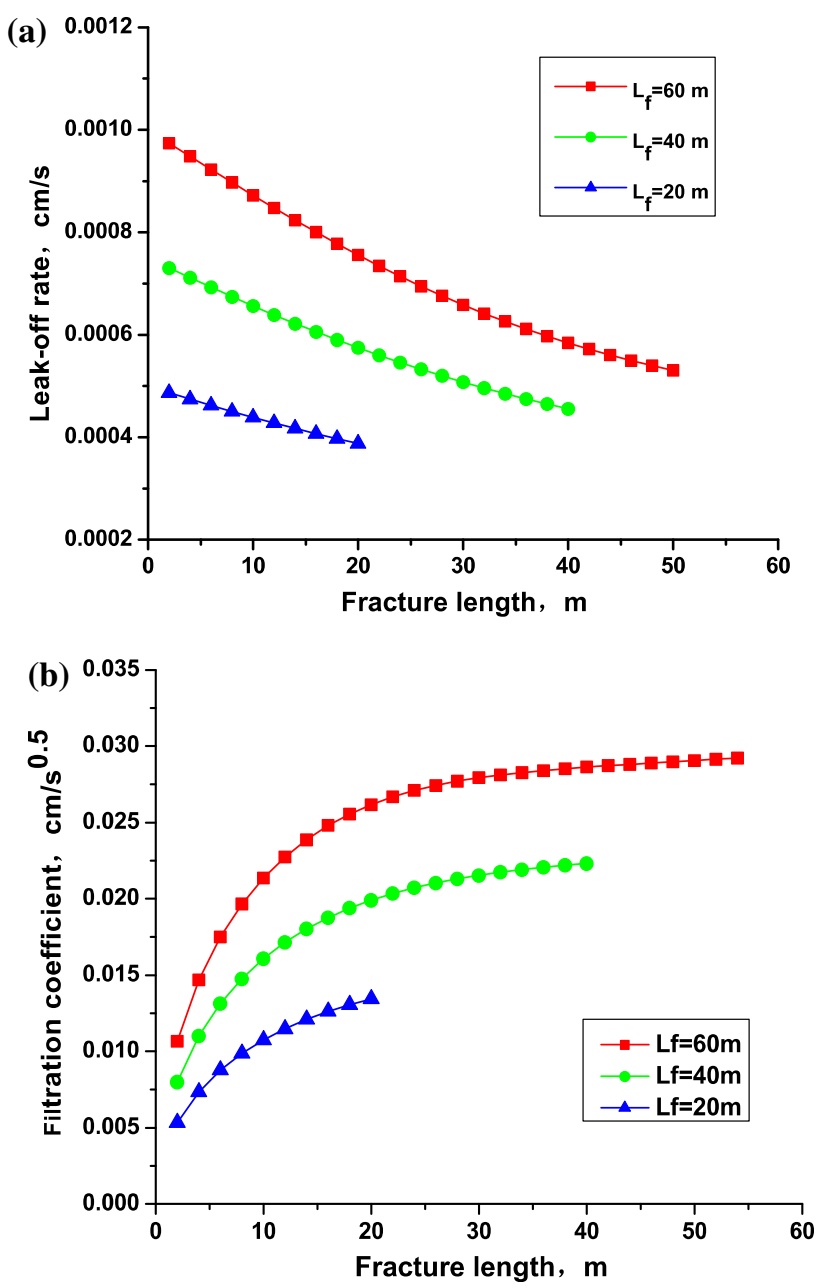

Fig. 6 The effect of different propagating lengths on fracturing fluid leak-off: a leak-off rate, b leak-off coefficient

effect results in the increase of the filtration coefficient later. Thus, an optimal displacement volume of fracturing fluid pumped in exists during the process of fracturing.

Figure 6 shows the laws of fracturing fluid leak-off considering the different stretching lengths of the artificial fractures. In Fig. 6, the lengths of the fractures are, respectively, 20, 40, and $60 \mathrm{~m}$. It can be concluded that, for a certain length of the fracture, the leak-off rate and the filtration coefficient are different, respectively, at the different locations of the fractures. The closer the distance to the end of the fracture is, the smaller the leak-off rate is and the greater the filtration coefficient is. For the different lengths of the fractures, the filtration coefficients are different at the same position of the fracture, the longer the fractures are, the greater the leak-off rate and the filtration coefficient are. Based on the analysis of the mechanism, the longer the fracture and the time of pumping in fracturing fluids are, the larger the artificial fracture pressure will be, and the amount of leak-off will also increase. While the 
closer to the end of the fracture, the more significant the effect of the sealed boundary will be, and the worse the formation pressure supply will be. All of the phenomena above will result in larger fracturing fluid leak-off rate and greater filtration coefficient.

\section{Conclusion}

1. Based on the classical PKN two-dimensional fracturestretching mathematical model, the two-dimensional leak-off model of fracturing fluid of fractured dualmedium reservoir is established by considering the time-varying non-Newtonian fracturing fluid leak-off coefficient in the stretching process of fractures;

2. Using the finite element difference method, a dynamic discrete grid system is established and solved by Newton-Raphson iterative method, and the relevant factors are analyzed;

3. As the stress sensitivity coefficient increases, the fracturing fluid filtration coefficient and fracturing fluid leak-off rate will decrease, while as the volume of fracturing fluid pumped in enlarges, the fracturing fluid filtration coefficient and the leak-off rate will increase. When the stress sensitivity coefficient or the displacement volume of fracturing fluid pumped in increases to a certain value, under the comprehensive effects of the formation pressure, the fracture pressure, the boundary, and the fracture permeability, the filtration coefficient will firstly decrease and then increase; and

4. Considering the propagation length of the fracture, the longer the length is, the more significant the leak-off effect will be, and the boundary effect close to the end of the fracture will increase the fracture filtration coefficient and the leak-off rate.

Acknowledgments The authors acknowledge a fund called the engineering theory and risk control of Deepwater drilling and completion from the National Basic Research Program of China (No. 2015CB251206) and supports from the MOE Key Laboratory of Petroleum Engineering.

Open Access This article is distributed under the terms of the Creative Commons Attribution 4.0 International License (http://creativecommons.org/licenses/by/4.0/), which permits unrestricted use, distribution, and reproduction in any medium, provided you give appropriate credit to the original author(s) and the source, provide a link to the Creative Commons license, and indicate if changes were made.

\section{References}

Al-Shatri A, Bellah S, Furuya K, Umezawa YT (2009) Successful application of 3D seismic technology on middle cretaceous carbonate reservoir-significant OIP Increasing. SPE paper 12539

Balen RM, Meng H-Z, Economides MJ (1988) Applications of the net present value (NPV) in the optimization of hydraulic fracture.SPE paper 18541

Demarchos AS, Chomatas AS (2004) Pushing the limits of hydraulic fracture design.SPE paper 86483

Economides MJ, Demarchos AS (2008) Benefits of a p-3D Over a 2D model for unified fracture design. SPE paper 112374

Fan Y, Economides MJ (1995) Fracturing fluid leak off and net pressure behavior in Frac and Pack stimulation. SPE paper29988

Gidley JL, Holditch SA, Nierode DE, Veatch Jr RW (1989) Recent advances in hydraulic fracturing. SPE Monogr, vol 12

Mayerhofer MJ, Economides MJ, Nolte KG (1991) An experimental and fundamental interpretation of fracturing filter-cake fluid loss. SPE paper 22873

Nghiem LX, Forsyth PA Jr, Behie A (1984) A fully implicit hydraulic fracture model. J Petroleum Technol 36(7):1191-1198

Ouenes ARC, Hartley LJ (2000) AEA Technology. Integrated fractured reservoir modeling using both discrete and continuum approaches. SPE paper 62939

Settari A (1985) A new general model of fluid loss in hydraulic fracturing. SPE-JPT pp 491-501

Settari A (1998) General model of fluid flow (leak off) from fractures induced in injection operations. SPE paper 18197

Yi TC, Penden JM (1993) A comprehensive model of fluid loss in hydraulic fracturing. Paper SPE 25493 